\title{
Impact of Persistent Antiphospholipid Antibodies on Risk of Incident Symptomatic Thromboembolism in Children: A Systematic Review and Meta-Analysis
}

\author{
Gili Kenet, M.D., 1 Sofia Aronis, M.D., ${ }^{2}$ Yackov Berkun, M.D., 3 \\ Mariana Bonduel, M.D., 4 Anthony Chan, M.D., 5 Neil A. Goldenberg, M.D., Ph.D., 6 \\ Susanne Holzhauer, M.D.,7 Alfonso lorio, M.D., 8 Janna Journeycake, M.D., 9 \\ Ralf Junker, M.D., ${ }^{10}$ Christoph Male, M.D., ${ }^{11}$ Marilyn Manco-Johnson, M.D., 5 \\ Patti Massicotte, M.D., ${ }^{12}$ Rolf Mesters, M.D., ${ }^{13}$ Paul Monagle, M.D., ${ }^{14}$ \\ Heleen van Ommen, M.D., ${ }^{15}$ Leslie Rafini, M.D., ${ }^{16}$ Paolo Simioni, M.D., 17 \\ Guy Young, M.D., ${ }^{18}$ and Ulrike Nowak-Göttl, M.D. ${ }^{10}$
}

\section{ABSTRACT}

The aim of this study was to estimate the impact of antiphospholipid (aPL) antibodies on the risk of incident thromboembolism (TE; arterial and venous) in children via meta-analysis of published observational studies. A systematic search of electronic databases (Medline, EMBASE, OVID, Web of Science, The Cochrane Library) for studies published from 1966 to 2010 was conducted using keywords in combination both as MeSH terms and text words. Two authors independently screened citations and those meeting the a priori defined inclusion criteria were retained. Data on year of publication, study design, country of origin, number of patients/controls, ethnicity, TE type, and frequency of recurrence were abstracted. Heterogeneity across studies was evaluated, and summary odds ratios (ORs) and 95\% confidence intervals (CIs) were calculated using either fixed-effects or random-effects models. Of 504, 16 pediatric studies met the inclusion criteria. In total 1403 patients and 1667 population-based controls $\leq 18$ years were enrolled. No significant heterogeneity was

\footnotetext{
${ }^{1}$ The Israel National Haemophilia Centre, Sheba Medical Centre, Tel-Hashomer, Israel; ${ }^{2}$ Haemophilia Centre-Haemostasis Unit, Aghia Sophia Children's Hospital, Athens, Greece; ${ }^{3}$ The Department of Pediatrics, Safra Children's Hospital, Sheba Medical Center, Tel-Hashomer, Israel; ${ }^{4}$ Servicio de Hematolgía y Oncología, Hospital de Pediatría "Prof. Dr. JP Garrahan," Buenos Aires, Argentina; ${ }^{5}$ Thrombosis and Atherosclerosis Research Institute McMaster University, Hamilton, Ontario, Canada; ${ }^{6}$ Department of Pediatrics and Medicine, Hematology/Oncology/BMT, University of Colorado and The Children's Hospital, Denver, Colorado; ${ }^{7}$ Department of Pediatric Hematology/Oncology, Charite, Berlin, Germany; ${ }^{8}$ Departments of Clinical Epidemiology \& Biostatistics and Medicine, McMaster University, Hamilton, Ontario, Canada; ${ }^{9}$ Department of Pediatrics, Children's Medical Centre Dallas, University of TX Southwestern, Medical Centre Dallas, Texas; ${ }^{10}$ Department of Clinical Chemistry, University Hospital of Kiel, Kiel, Germany; ${ }^{11}$ Department for Pediatrics, Medical University Vienna, Vienna, Austria; ${ }^{12}$ Department of Pediatrics, University Children Hospital Alberta, Canada; ${ }^{13}$ Department of Internal Medicine, University Hospital of Münster, Münster, Germany; ${ }^{14}$ The Royal Children's Hospital, Victoria, Australia; ${ }^{15}$ Department of Pe-
}

diatric Hematology, Emma Children's Hospital AMC, Amsterdam, The Netherlands; ${ }^{16}$ Division of Hematology, Department of Pediatrics, The Children's Hospital of Philadelphia, University of Pennsylvania School of Medicine, Philadelphia, Pennsylvania; ${ }^{17}$ Department of Medical \& Surgical Sciences, University of Padua, Padua, Italy; ${ }^{18}$ Division of Hematology/Oncology, Children's Hospital Los Angeles, Los Angeles, California.

Address for correspondence and reprint requests: Ulrike NowakGöttl, M.D., Hemostasis Unit, Department of Clinical Chemistry, University Hospital of Kiel, Arnold-Heller-Str. 3, D-24105 Kiel, Germany (e-mail: leagottl@gmail.com).

Hemostasis and Thrombosis in Pediatric Patients: Special Issues and Unique Concerns; Guest Editors, Gili Kenet, M.D., and Ulrike Nowak-Göttl, M.D.

Semin Thromb Hemost 2011;37:802-809. Copyright (C) 2011 by Thieme Medical Publishers, Inc., 333 Seventh Avenue, New York, NY 10001, USA. Tel: +1(212) 584-4662.

DOI: http://dx.doi.org/10.1055/s-0031-1297171.

ISSN 0094-6176. 
discerned across studies, and no publication bias was detected. Thus, data from arterial and venous $\mathrm{TE}$ were analyzed together. In addition, meta-regression analysis did not reveal statistically significant differences between site of TE, age at first TE, country, or publication year. A statistically significant association with a first TE was demonstrated for persistent aPL antibodies, with an overall summary ORs/CI of 5.9/3.6-9.7 (arterial 6.6/3.5-12.4; deep vein thrombosis 4.9/2.2-10.9). The present meta-analysis indicates that detection of persistent aPL is clinically meaningful in children with, or at risk for, TE and underscores the importance of pediatric thrombophilia screening programs.

KEYWORDS: Antiphospholipid antibodies, thromboembolism, recurrence, children, meta-analysis

Antiphospholipid syndrome (APS) is a multisystem autoimmune disease, characterized by arterial and/or venous thrombosis, recurrent fetal loss, and persistent circulating antiphospholipid (aPL) antibodies. ${ }^{1}$ This syndrome can be either primary or secondary to other autoimmune diseases, with 9 to $14 \%$ in children with systemic lupus erythematosus. ${ }^{2-4}$

Pediatric primary APS is very rare, and disease onset before age 15 occurred in only $2.8 \%$ of patients from the largest published/reported APS cohort. $^{5-27}$

Adapted from the adult definition, pediatric APS has been defined as one or more arterial or venous thrombosis associated with persistent aPL antibodies, that is, immunoglobulin M (IgM) or immunoglobulin $\mathrm{G}$ (IgG), anticardiolipin (ACL), anti beta-2 glycoprotein 1 antibodies, ${ }^{28}$ or the presence of lupus anticoagulants, summed up as antiphospholipid antibodies (APLA), confirmed in at least one follow-up examination more than 12 weeks apart. ${ }^{29}$

It is obvious that obstetric morbidity, the first of the two diagnostic clinical criterion, is not applicable in children, and as far as vascular thrombosis is concerned, the second criterion, the children are less prone to it due to rarity of additional thrombophilic risk factors such as atherosclerosis and use of oral contraceptives in this age range. On the other hand, many important and common manifestations of the syndrome are not included, such as livedo reticularis, chorea, and thrombocytopenia. However, the same criteria are used for classification of pediatric APS without any validation in children. ${ }^{29}$ In front of the diagnostic challenge presented by aPL syndrome in childhood, a correct and early diagnosis of this risk condition is likely to produce striking benefit in term of morbidity. In fact, aPL plays an important role in the pathogenesis of pediatric thromboembolism (TE).$^{6-8}$ Interestingly, arterial TE or stroke are more often associated with primary APS as compared with deep venous thrombosis (DVT), which is observed predominantly in children with secondary APS. ${ }^{6}$ Nevertheless, results of single studies on the risk of TE onset associated with APS have been contradictory or inconclusive, mainly due to lack of statistical power. ${ }^{7-27}$ The aim of this article and systematic meta-analysis on published observational studies was to estimate the impact of APS on risk of childhood arterial and venous TE.

\section{METHODS}

The present systematic review and meta-analysis was performed in accordance with Strengthening the Reporting of Observational Studies in Epidemiology (STROBE) guidelines recently published, ${ }^{30}$ with adaptations as further described below.

\section{Inclusion/Exclusion Criteria}

Published studies of TE in children $\leq 18$ years of age from 1966 through August 2010 were evaluated for inclusion if (1) aPL antibodies or the presence of lupus anticoagulants were individually investigated in a given TE cohort (descriptive analysis), and if (2) the frequency of aPL was compared between TE patients and control subjects without history of TE in a given study (meta-analysis). Pediatric TE events of any location objectively confirmed by suitable imaging methods were included. In addition, for inclusion, publications must have reported the country of origin, study design, ethnicity, numbers of patients/controls, type of TE (arterial vs. venous), number of individuals tested for aPL, screening tests performed, and laboratory methods (including criteria for normal/abnormal results). For the purposes of the present work, a classification of arterial TE was used to include both peripheral arterial thrombosis as well as arterial ischemic stroke. Case reports and case series/studies in which less than $50 \%$ of cases were systematically screened for aPL, publications reporting asymptomatic thrombosis, and studies with unclear laboratory/analytical methodology to differentiate between transient and persistent aPL were not included (except in instances wherein this distinction was subsequently clarified in personal communication with the responsible authors; only cases with inherited deficiency states were included in the analyses). 


\section{Search Strategy}

A systematic search of publications listed in the electronic databases (Medline via PubMed, EMBASE, OVID, Web of Science, The Cochrane Library) from 1970 to August 2010 was conducted using the following keywords in combination both as $\mathrm{MeSH}$ terms and text words: ("arterial thrombosis" OR "stroke" OR "deep vein thrombosis" OR "thromboembolism" OR "venous thromboembolism" OR "pulmonary embolism" OR "renal venous thrombosis" OR "cerebral venous thrombosis") AND ("neonate" OR "infant" OR "children" OR "child" OR "childhood" OR "adolescents" OR "pediatric" OR "paediatric" NOT “adult") AND ("thrombophilia" OR "prothrombotic" OR "procoagulant" OR "antiphospholipid antibodies" OR "lupus anticoagulant" OR "anticardiolipin antibodies" OR "antibodies"). In addition, reference lists of journal articles identified through the aforementioned search were then manually searched to locate additional studies. The search strategy had no language restrictions. Citations were screened and classified into cohort/case-control, case series, or registry data by two independent group members (A.C. and H.v.O.). Those meeting the inclusion criteria were retained. The decision to include or exclude studies was hierarchical, initially made on the basis of the study title, followed by the abstract, and finally the complete body text. In the event of conflicting opinions, resolution was achieved through discussion.

\section{Data Extraction}

To avoid possible double counting of patients included in more than one report of the same authors/working groups, the patient recruitment periods and catchment areas were evaluated and authors were contacted for clarification. If the required data could not be located in the published report, the corresponding author was contacted to provide the missing data of interest. Multiple reviewers checked data extractions for accuracy.

\section{Study Design Classification}

In this meta-analysis, studies were classified as having a "cohort" design when subjects with and without a factor being investigated were followed up for development of the outcome of interest. Studies were classified as having a "case-control" design when individuals grouped by outcome (i.e., cases having the outcome/disease of interest and controls without the outcome/disease) were compared for the presence of a risk factor of interest. A "case report" or "case series" was defined as one case or a group of cases of a particular outcome of interest with no control group. Studies were classified as having a "registry" design when consisting of a multicenter populationbased (ascertainment of $>90 \%$ of cases available) case series employing predefined standardized data collection criteria, but no requirement for longitudinal follow-up data collection. ${ }^{31}$

\section{Missing Data}

Studies in which symptomatic pediatric TE patients were not screened for aPL or studies in which the aPL screening was $<50 \%$ of cases, or was performed in sporadic cases only were not included in the present meta-analysis. When the percentage of patients screened for aPL was not clear from the manuscript, the authors were asked for clarification.

\section{Statistical Analyses}

Data analyses were performed using STATA version 9 (StataCorp LP, College Station, TX), StatsDirect version 2.6.6 (StatsDirect Ltd., Altrincham, Cheshire, UK) and Comprehensive Meta-analysis version 2.2.046 (Biostat Inc., Englewood, NJ). Continuous data are presented as median (minimum-maximum: min-max) values. For meta-analysis, summary ORs and $95 \% \mathrm{CIs}$ were calculated from the effect estimates of the individual studies weighted by standard error using both a fixed-effects model (weighting each estimate by its standard error, via the Mantel and Haenszel method) and a random-effects model (estimating between-study variance in effect measures according to DerSimonian and Laird $)^{32}$; the latter approach was used to control for heterogeneity, according to Higgins et al. ${ }^{33}$ aPL were analyzed together and compared with the absence of aPL as reference category. A $p$ value $<0.05$ was considered statistically significant. In addition, meta-regression analysis was performed to evaluate statistical significant differences between locations of TE, age at first thromboembolic event, country, or publication year. Heterogeneity among studies was assessed using $\mathrm{I}^{2}$ statistics: when $p<0.05$, presence of heterogeneity was considered statistically significant, and when $\mathrm{I}^{2}>50 \%$, magnitude of heterogeneity was considered substantial. ${ }^{34}$ Funnel plots of effect size against standard error and a modified linear regression test were used to describe the presence of publication bias. The authors had full access to and take full responsibility for the integrity of the data. All authors have read and agreed to the manuscript as written.

\section{RESULTS}

\section{Descriptive Analyses}

From 504 potentially relevant citations ascertained from electronic databases and search of reference lists, 16 cohort/case-control studies or case series/registries from 7 countries finally met the inclusion criteria for descriptive analysis (Table 1; Fig. 1). Beginning in 
Table 1 Studies Qualifying for Analysis

\begin{tabular}{|c|c|c|c|c|}
\hline Author and Year & TE Site & $\begin{array}{l}\text { Median Age at } \\
\text { TE Onset (Years) }\end{array}$ & $\begin{array}{l}\text { Persistent APLA } \\
\text { Positive (Cases) }\end{array}$ & $\begin{array}{l}\text { Persistent APLA } \\
\text { Positive (Controls) }\end{array}$ \\
\hline Nowak-Göttl et al, 1999 & Arterial & 5 & $1 / 148$ & $2 / 296$ \\
\hline Sträter et al, 1999 & Arterial & 6 & $4 / 38$ & $1 / 182$ \\
\hline Günther et al, 2000 & Arterial & 0,1 & $4 / 91$ & Ref. Sträter et al, 1999 \\
\hline Kenet et al, 2000 & Arterial & 5,6 & $9 / 58$ & $3 / 89$ \\
\hline Kurnik et al, 2003 & Arterial & 0,1 & $15 / 125$ & Ref. Sträter et al, 1999 \\
\hline Duran et al, 2005 & Arterial & 2,5 & $5 / 30$ & $2 / 33$ \\
\hline Bonduel et al, 2006 & Arterial & 8 & $5 / 112$ & $1 / 212$ \\
\hline Nowak-Göttl et al, 2008 & Arterial & 7 & $4 / 282$ & Ref. Sträter et al, 1999 \\
\hline Simchen et al, 2009 & Arterial & 0,1 & $5 / 23$ & $5 / 100$ \\
\hline Heller et al, 2000 & Venous & 0,1 & $4 / 34$ & $2 / 100$ \\
\hline Heller et al, 2003 & Venous & 6 & $6 / 149$ & $1 / 149$ \\
\hline Kenet et al, 2004 & Venous & 5,6 & $4 / 38$ & $2 / 112$ \\
\hline Kosch et al, 2004 & Venous & 0,1 & $3 / 59$ & $1 / 118$ \\
\hline Gürgey et al, 2004 & Venous & 14 & $2 / 46$ & $1 / 87$ \\
\hline Ünal et al, 2005 & Venous & 8,5 & $1 / 37$ & Ref. Gürgey et al, 2004 \\
\hline Bonduel et al, 2006 & Venous & 2,0 & $2 / 110$ & $1 / 102$ \\
\hline
\end{tabular}

TE, thromboembolism; APLA, antiphospholipid antibodies.

1999, the presence of aPL was systematically investigated in childhood TE (nine studies in arterial TE and seven studies in pediatric index cases with venous TE). The median (min-max) age at onset in studies reporting $\mathrm{TE}$ was 5.3 years $(0.1$ to 14.5$)$ and in 74 of 1380 patients (5.4\%), persistent aPL were diagnosed. Of note, due to author's clarification individual numbers of patients and controls in the studies listed in tables may vary from original study manuscripts.

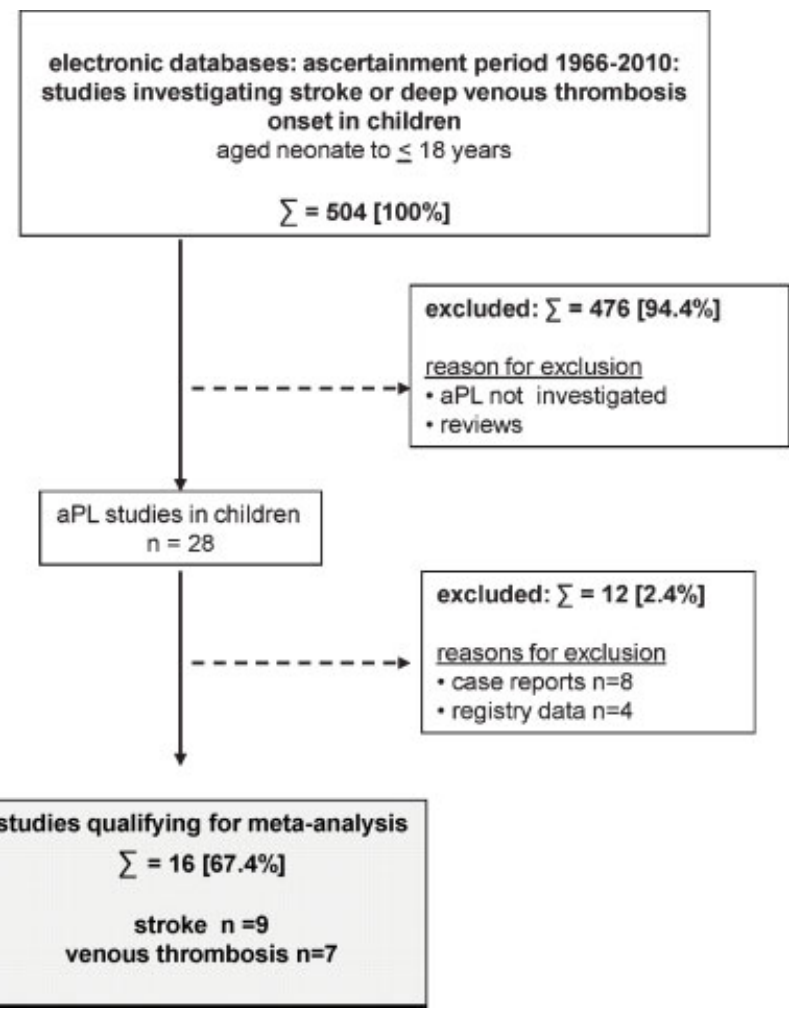

Figure 1 Study flow chart. 
Table 2 Summary (ORs/95\%Cls: Meta-Analysis) Including Testing for Heterogeneity $\left(I^{2}\right)$, and Publication Bias for Persistent APLA Associated with a First TE Onset in Children

\begin{tabular}{|c|c|c|c|}
\hline & $\begin{array}{l}\text { ORs } / 95 \% \mathrm{Cl} ; \\
\text { Fixed Model }\end{array}$ & $I^{2}(\%), p$ Value & $\begin{array}{l}\text { Bias Indicator } \\
\text { (Harbord) }\end{array}$ \\
\hline Arterial TE $(n=9)$ & $6.6 / 3.5-12.4$ & - & - \\
\hline venous TE $(n=7)$ & $4.9 / 2.2-10.9$ & - & - \\
\hline Combined TE $(n=23)$ & $5.9 / 3.6-9.7$ & $0, p=0.93$ & $p=0.32$ \\
\hline
\end{tabular}

ORs, odds ratios; Cl: confidence intervals; APLA, antiphospholipid antibodies; TE, thromboembolism.

\section{Meta-Analyses}

Table 2 summarizes the number of studies and patients included in the meta-analysis. As no significant heterogeneity was discerned across studies, summary ORs and 95\% CIs under a fixed-effects model are shown. Summary ORs were calculated separately by TE type (arterial vs. venous events or DVT), and then pooled across: since heterogeneity assessment did not reveal any statistically significant effect of locations of TE type. Further, metaregression did not reveal any effect of age at first thromboembolic event (Fig. 2), study country or publication year, pooled summary ORs were preferred. As depicted in the Forest plot of Fig. 3, a statistically significant association with a first TE during childhood was demonstrated for the presence of persistent APLA, with an overall summary OR/CI of 5.9/3.6-9.7 (arterial TE: 6.6/3.5-12.4; venous TE: 4.9/2.2-10.9). Fig. 4 depicts the funnel plot of effect size against standard error for each study investigated, which was broadly symmetrical and consistent with the conclusion that there was no major publication bias.

\section{DISCUSSION}

Apart from registry data on the role of aPL in children with $\mathrm{TE}$ to the best of our knowledge this is the first meta-analysis on observational studies investigating the association of aPL and TE in children including com-

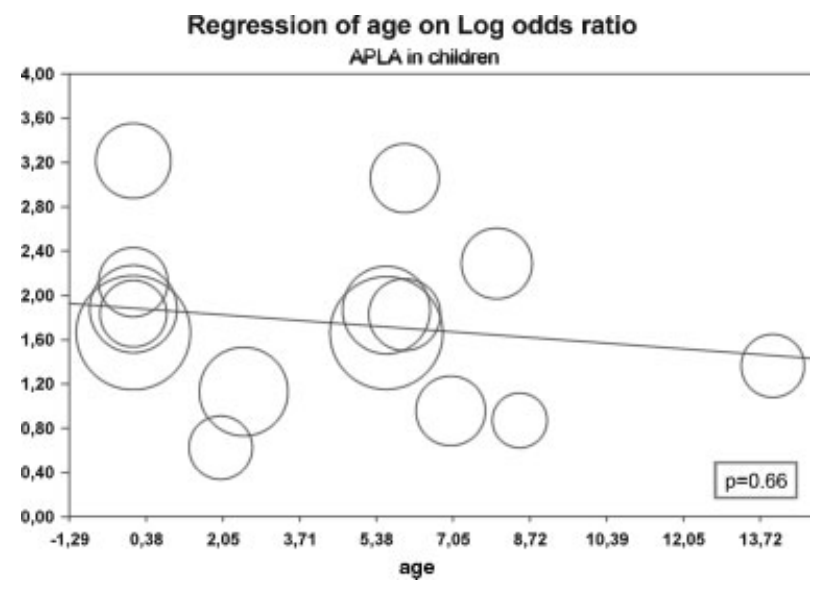

Figure 2 Regression analysis depicting the association between age at onset and persistent APLA in children with TE. APLA, antiphospholipid antibodies; TE, thromboembolism. bining recent data from children with stroke. The presence of aPL show a significant association with first onset of pediatric TE, with the highest ORs found for arterial thrombosis or stroke. Our findings are concordant with data from the international pediatric APS registry, ${ }^{6,35}$ however, vary in the fact that we could not find a different age distribution between types of TE in regression analysis.

In an Israeli study including 28 pediatric APS patients it was shown that the thrombotic event may be spontaneous or due to predisposing factor, such as vascular stasis, trauma, surgery, use of oral contraceptives, and inherited thrombophilia. DVT of the lower limbs was the commonest thrombosis in pediatric APS patients in the Israeli cohort. A high rate of progression to lupus in girls with primary APS was found in that cohort, yet neither renal nor any heart and skin disease appeared during follow-up. Recurrent thrombotic events were less frequent in patients who received anticoagulant therapy. The study also found a high rate of inherited thrombophilias ( $45 \%$ of patients) and proposed that aPL may serve as a "second vascular hit" in children, thus thromboses tended to recur if proper long-term anticoagulation was not applied. ${ }^{7}$ The recently published pediatric APS registry reported earlier thrombotic manifestations in children with primary versus secondary APS with a similar gender distribution. In this retrospective registry $60 \%$ of the thromboembolic events reported were of venous thrombosis and thrombosis of arterial origin reported in $32 \%$ of cases were mainly pediatric stroke. ${ }^{35}$

This project of an international registry of pediatric patients with APS was initiated in 2004 to foster and conduct multicenter, controlled studies with large number of pediatric APS patients. The Ped-APS Registry was organized as a collaborative project of the European Forum on aPL and Juvenile Systemic Lupus Erythematosus Working Group of the Pediatric Rheumatology European Society. ${ }^{36}$ Currently, it documented a standardized clinical, laboratory, and therapeutic data of 133 children with aPL-related thrombosis from 14 countries.

In addition to symptomatic TE reported in infants, children, and adolescents, rare cases of perinatal thrombosis in infants born to mothers with APS or aPL have been reported. ${ }^{37,38}$ In this neonatal registry positive aPL tests were observed in most infants and the clinical 
Meta Analysis APLA in children

\begin{tabular}{|c|c|c|c|c|c|c|}
\hline \multirow[t]{2}{*}{ Author-year } & \multirow[t]{2}{*}{ Subgroup within study } & \multicolumn{3}{|c|}{ Cumulative statistics } & & \multirow[t]{2}{*}{ Cumulative odds ratio $(95 \% \mathrm{Cl}$} \\
\hline & & $\begin{array}{l}\text { Lower } \\
\text { limit }\end{array}$ & $\begin{array}{l}\text { Upper } \\
\text { limit }\end{array}$ & Z-Value & -Value & \\
\hline Strater 1999 & 21,294 & 2,309 & 196,399 & 2,698 & 0,007 & \\
\hline Günther 2000 & 13,268 & 2,773 & 63,486 & 3,237 & 0,001 & \\
\hline Kenet 2000 & 7,817 & 2,809 & 21,756 & 3,937 & 0,000 & \\
\hline Kurnik 2003 & 9,872 & 3,955 & 24,642 & 4,906 & 0,000 & \\
\hline Duran 2005 & 7,651 & 3,411 & 17,163 & 4,937 & 0,000 & \\
\hline Bonduel 2006 & 7,893 & 3,704 & 16,822 & 5,352 & 0,000 & \\
\hline Nowak-Gottl 200 & 7,019 & 3,432 & 14,356 & 5,338 & 0,000 & \\
\hline Simchen 2008 & 6,588 & 3,505 & 12,382 & 5,856 & 0,000 & \\
\hline Heller 2000 & 6,582 & 3,636 & 11,914 & 6,224 & 0,000 & \\
\hline Heller 2003 & 6,554 & 3,701 & 11,608 & 6,446 & 0,000 & \\
\hline Kenet 2004 & 6,546 & 3,803 & 11,268 & 6,781 & 0,000 & \\
\hline Kosch 2004 & 6,531 & 3,850 & 11,077 & 6,961 & 0,000 & s \\
\hline Gurgey 2004 & 6,381 & 3,808 & 10,693 & 7,036 & 0,000 & \\
\hline Unal 2005 & 6,178 & 3,718 & 10,265 & 7,030 & 0,000 & \\
\hline \multirow[t]{2}{*}{ Bonduel 2006b } & 5.873 & 3.574 & 9.653 & 6.984 & 0.000 & \\
\hline & 5,873 & 3,574 & 9,653 & 6,984 & 0,000 & \\
\hline
\end{tabular}

Figure 3 Cumulative Forest plot shows odds ratios and 95\% confidence intervals (Cls) for observational studies investigating the influence of persistent antiphospholipid antibodies on first onset of symptomatic TE in children. The study author and year of publication are indicated on the $y$ axis. The box for each study is proportional to the inverse of variance; horizontal lines show the $95 \% \mathrm{Cl}$ of the ORs. The pooled estimate is based on a random effects model shown by a vertical line and diamond (95\% $\mathrm{Cl}$ ). Studies are listed in descending order by year of publication. Studies with patients/controls events counted as "zero" are not depicted.

presentation consisted of arterial and venous thromboses in multiple localizations being similar to adult APS patients. The contribution of aPL to the multifactorial nature of perinatal arterial stroke is not yet well defined. In a mother-infant cohort study, ${ }^{27}$ evidence of aPL antibodies, in either mother or child was significant. More than one-fifth of perinatal stroke infants in this study tested positive for aPL, and aPL was found in $18.2 \%$ of mothers compared with $4.7 \%$ of control mothers.
Although the clinical and laboratory manifestations of this subgroup are consistent with the diagnostic criteria of APS, their disease behaves differently and rarely recurs, as opposed to thromboembolic events in older children with APS. ${ }^{35}$ Thus, perinatal stroke in children with APS deserves special consideration and may not require anticoagulant therapy unless other risk factors prevail.

The current literature review and meta-analysis clearly show that pediatric APS plays an important role

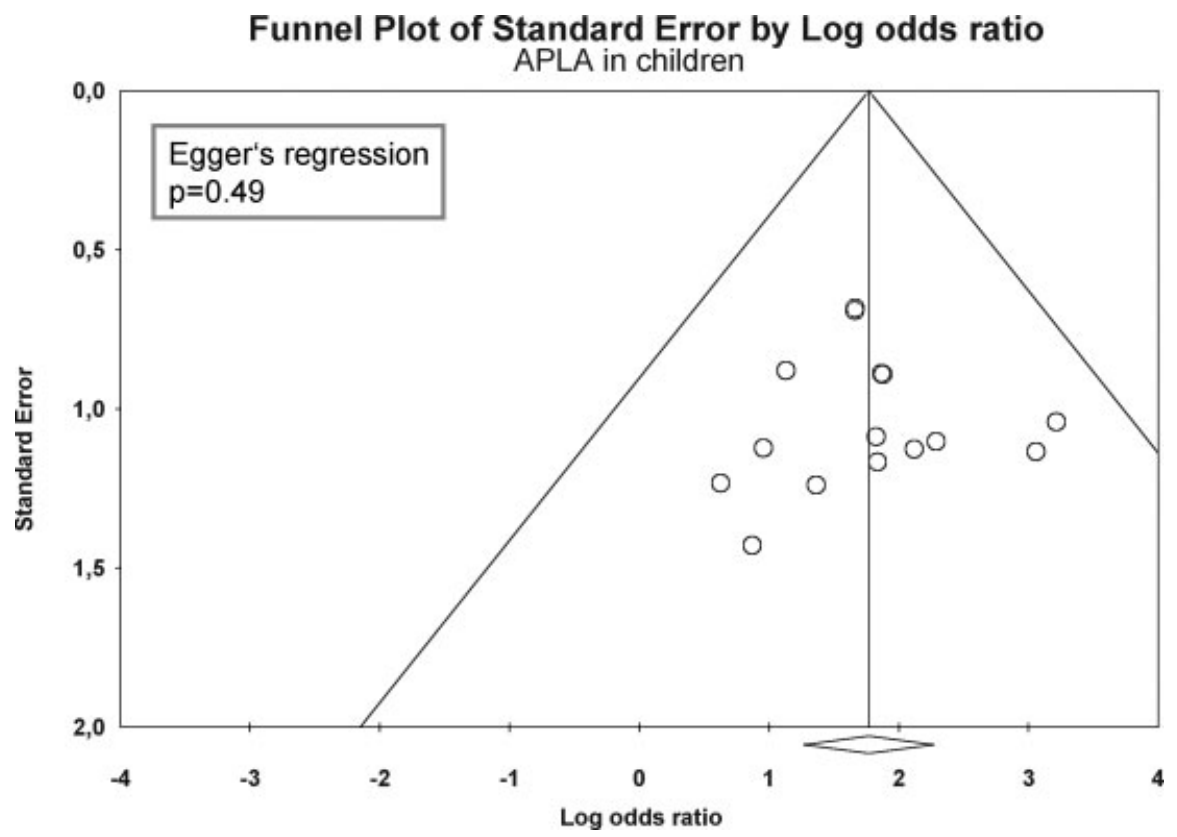

Figure 4 Depicts the funnel plot of effect size against standard error for each study investigated and was broadly symmetrical, which was consistent with the conclusion that there was no major publication bias. 
among thrombophilic risk factors for thrombosis in young infants and children.

Limitations of the present meta-analysis are several. First, the included studies were conducted over different time periods; therefore it has to be taken into consideration that diagnosis and treatment modalities including referral patterns might have changed over time. Second, most of the studies included in our meta-analysis were undertaken in white children with TE, thus it is unknown if our findings can be extrapolated to other ethnic groups. Third, although formal testing did not show a publication bias it cannot completely ruled out considering the rarity of the disease and the small number of studies available in the field of pediatric TE. Fourth, given the relatively small number of infants included in the original studies and hence this meta-analysis, its findings may not be able to be extended to this age group.

Notwithstanding these limitations, the findings of the present meta-analysis that persistent aPL is associated with $\mathrm{TE}$ onset in children have important implications for future advancement of the field. The results of this meta-analysis suggest that future investigation of secondary prevention in pediatric venous $\mathrm{TE}$ should include trials of prolonged anticoagulant treatment duration targeting high-risk inherited thrombophilia groups, as well as study of primary prophylaxis against TE in highrisk situations among children without history of TE who are identified to have persistent aPL antibodies.

\section{ACKNOWLEDGMENTS}

We thank Klaus Berger for helping in epidemiologic discussions, and Sarah Nixdorf and Doris Kunkel for technical support.

\section{NOTES}

Funding Sources: The study was supported by grants from the "Förderverein Schlaganfall und Thrombosen im Kindesalter e.V." The study supporters had no role in study design, data collection, data analysis, data interpretation, or in writing of the report.

Authors' Contributions: Along with the principal study investigators, that is, Gili Kenet, Neil Goldenberg, and Ulrike Nowak-Göttl who act as the guarantors, all other investigators had full access to the data (Sofia Aronis, Yackov Berkun, Mariana Bonduel, Anthony Chan, Susanne Holzhauer, Alfonso Iorio, Janna Journeycake, Ralf Junker, Christoph Male, Marilyn Manco-Johnson, Patti Massicotte, Rolf Mesters, Paul Monagle, Heleen van Ommen, Leslie Raffini, Paolo Simioni, Guy Young) and took part in the design, execution and data analysis, and in writing the report. Alfonso Iorio and Ulrike Nowak-Göttl were responsible for the statistical calculation.

\section{REFERENCES}

1. Miyakis $\mathrm{S}$, Lockshin MD, Atsumi $\mathrm{T}$, et al. International consensus statement on an update of the classification criteria for definite antiphospholipid syndrome (APS). J Thromb Haemost 2006;4(2):295-306

2. Levine JS, Branch DW, Rauch J. The antiphospholipid syndrome. N Engl J Med 2002;346(10):752-763

3. Levy DM, Massicotte MP, Harvey E, Hebert D, Silverman ED. Thromboembolism in paediatric lupus patients. Lupus 2003;12(10):741-746

4. Campos LM, Kiss MH, D'Amico EA, Silva CA. Antiphospholipid antibodies and antiphospholipid syndrome in 57 children and adolescents with systemic lupus erythematosus. Lupus 2003;12(11):820-826

5. Cervera R, Piette JC, Font J, et al; Euro-Phospholipid Project Group. Antiphospholipid syndrome: clinical and immunologic manifestations and patterns of disease expression in a cohort of 1,000 patients. Arthritis Rheum 2002; 46(4):1019-1027

6. Avcin T, Cimaz R, Rozman B; Ped-APS Registry Collaborative Group. The Ped-APS Registry: the antiphospholipid syndrome in childhood. Lupus 2009;18(10):894-899

7. Berkun Y, Padeh S, Barash J, et al. Antiphospholipid syndrome and recurrent thrombosis in children. Arthritis Rheum 2006;55(6):850-855

8. Kenet G, Lütkhoff LK, Albisetti M, et al. Impact of inherited thrombophilia on arterial ischemic stroke and cerebral sinovenous thrombosis in children: a systematic review and meta-analysis of observational studies. Circulation 2010;121:1838-1847

9. Male C, Mitchell L, Julian J, et al. Acquired activated protein $\mathrm{C}$ resistance is associated with lupus anticoagulants and thrombotic events in pediatric patients with systemic lupus erythematosus. Blood 2001;97(4):844-849

10. Levy DM, Massicotte MP, Harvey E, Hebert D, Silverman ED. Thromboembolism in paediatric lupus patients. Lupus 2003;12(10):741-746

11. Bonduel M, Sciuccati G, Hepner M, et al. Factor V Leiden and prothrombin gene G20210A mutation in children with cerebral thromboembolism. Am J Hematol 2003;73(2): 81-86

12. Günes AM, Baytan B, Günay U. The influence of risk factors in promoting thrombosis during childhood: the role of acquired factors. Pediatr Hematol Oncol 2006;23(5): 399-410

13. Nowak-Göttl U, Junker R, Hartmeier M, et al. Increased lipoprotein(a) is an important risk factor for venous thromboembolism in childhood. Circulation 1999a;100 (7):743-748

14. Sträter R, Vielhaber H, Kassenböhmer R, von Kries R, Göbel U, Nowak-Göttl U. Genetic risk factors of thrombophilia in ischaemic childhood stroke of cardiac origin. A prospective ESPED survey. Eur J Pediatr 1999;158(Suppl 3): S122-S125

15. Heller C, Schobess R, Kurnik K, et al; For the Childhood Thrombophilia Study Group. Abdominal venous thrombosis in neonates and infants: role of prothrombotic risk factors - a multicentre case-control study. Br J Haematol 2000; 111(2): 534-539

16. Günther G, Junker R, Sträter R, et al; Childhood Stroke Study Group. Symptomatic ischemic stroke in full-term neonates : role of acquired and genetic prothrombotic risk factors. Stroke 2000;31(10):2437-2441 
17. Kenet G, Sadetzki S, Murad H, et al. Factor V Leiden and antiphospholipid antibodies are significant risk factors for ischemic stroke in children. Stroke 2000;31(6): 1283-1288

18. Heller C, Heinecke A, Junker R, et al; Childhood Stroke Study Group. Cerebral venous thrombosis in children: a multifactorial origin. Circulation 2003;108(11):1362-1367

19. Kurnik K, Kosch A, Sträter R, Schobess R, Heller C, Nowak-Göttl U; Childhood Stroke Study Group. Recurrent thromboembolism in infants and children suffering from symptomatic neonatal arterial stroke: a prospective follow-up study. Stroke 2003;34(12):2887-2892

20. Gürgey A, Balta G, Gumruk F, Altay C. Analysis of some clinical and laboratory aspects of adolescent patients with thrombosis. Blood Coagul Fibrinolysis 2004;15(8): 657-662

21. Kenet G, Waldman D, Lubetsky A, et al. Paediatric cerebral sinus vein thrombosis. A multi-center, case-controlled study. Thromb Haemost 2004;92(4):713-718

22. Kosch A, Kuwertz-Bröking E, Heller C, Kurnik K, Schobess R, Nowak-Göttl U. Renal venous thrombosis in neonates: prothrombotic risk factors and long-term follow-up. Blood 2004;104(5):1356-1360

23. Duran R, Biner B, Demir M, Celtik C, Karasalihoğlu S. Factor $\mathrm{V}$ Leiden mutation and other thrombophilia markers in childhood ischemic stroke. Clin Appl Thromb Hemost 2005;11(1):83-88

24. Ünal S, Varan A, Yalçin B, Büyükpamukçu M, Gürgey A. Evaluation of thrombotic children with malignancy. Ann Hematol 2005;84(6):395-399

25. Bonduel M, Sciuccati G, Hepner M, et al. Arterial ischemic stroke and cerebral venous thrombosis in children: a 12 -year Argentinean registry. Acta Haematol 2006;115(3-4): 180-185

26. Nowak-Göttl U, Langer C, Bergs S, Thedieck S, Sträter R, Stoll M. Genetics of hemostasis: differential effects of heritability and household components influencing lipid concentrations and clotting factor levels in 282 pediatric stroke families. Environ Health Perspect 2008;116(6):839-843
27. Simchen MJ, Goldstein G, Lubetsky A, Strauss T, Schiff E, Kenet G. Factor v Leiden and antiphospholipid antibodies in either mothers or infants increase the risk for perinatal arterial ischemic stroke. Stroke 2009;40(1):65-70

28. Avcin T, Ambrozic A, Kuhar M, Kveder T, Rozman B. Anticardiolipin and $\beta 2$ glycoprotein I antibodies in sera of 61 apparently healthy children at regular preventive visits. Rheumatology 2001;40:555-573

29. Berkun Y, Kenet G. Pediatric antiphospholipid syndrome. Isr Med Assoc J 2008;10(1):45-47

30. Vandenbroucke JP, von Elm E, Altman DG, et al; STROBE initiative. Strengthening the reporting of observational studies in epidemiology (STROBE): explanation and elaboration. Ann Intern Med 2007;147(8):W163-94

31. Mayer D. Essential Evidence-Based Medicine. UK, Cambridge University Press; 2004:347-360

32. Kirkwood BR, Sterne JAC. Medical Statistics. 2nd ed. Oxford, Blackwell Science; 2003:180-387

33. Higgins JPT, Thompson SG, Deeks JJ, Altman DG. Measuring inconsistency in meta-analyses. BMJ 2003;327 (7414):557-560

34. Harbord RM, Egger M, Sterne JAC. A modified test for small-study effects in meta-analyses of controlled trials with binary endpoints. Stat Med 2006;25(20):3443-3457

35. Avčin T, Cimaz R, Silverman ED, et al. Pediatric antiphospholipid syndrome: clinical and immunologic features of 121 patients in an international registry. Pediatrics 2008;122 (5):e1100-e1107

36. Avcin T, Cimaz R, Meroni PL. Recent advances in antiphospholipid antibodies and antiphospholipid syndromes in pediatric populations. Lupus 2002;11(1):4-10

37. Boffa MCAM, Aurousseau MH, Lachassinne E, et al. European register of babies born to mothers with antiphospholipid syndrome. Lupus 2004;13(9):713-717

38. Boffa MC, Lachassinne E. Infant perinatal thrombosis and antiphospholipid antibodies: a review. Lupus 2007;16(8): 634-641 